\title{
Statistical description of rotating Kaluza-Klein black holes
}

\author{
Roberto Emparan ${ }^{1,2, *}$ and Alessandro Maccarrone ${ }^{2, \dagger}$ \\ ${ }^{1}$ Institució Catalana de Recerca i Estudis Avançats (ICREA) \\ ${ }^{2}$ Departament de Física Fonamental, Universitat de Barcelona, Diagonal 647, E-08028 Barcelona, Spain
}

(Received 1 February 2007; published 5 April 2007)

\begin{abstract}
We extend the recent microscopic analysis of extremal dyonic Kaluza-Klein (D0-D6) black holes to cover the regime of fast rotation in addition to slow rotation. Fastly rotating black holes, in contrast to slow ones, have nonzero angular velocity and possess ergospheres, so they are more similar to the Kerr black hole. The D-brane model reproduces their entropy exactly, but the mass gets renormalized from weak to strong coupling, in agreement with recent macroscopic analyses of rotating attractors. We discuss how the existence of the ergosphere and superradiance manifest themselves within the microscopic model. In addition, we show in full generality how Myers-Perry black holes are obtained as a limit of Kaluza-Klein black holes, and discuss the slow and fast rotation regimes and superradiance in this context.
\end{abstract}

DOI: 10.1103/PhysRevD.75.084006

PACS numbers: 04.70.Dy, 11.25.Uv

\section{INTRODUCTION}

Rotating black holes with maximal angular momentum provide an interesting setting for the investigation of black hole microphysics. Consider the intriguingly simple form of the entropy of the extremal Kerr black hole,

$$
S=2 \pi|J|
$$

Since the angular momentum $J$ is naturally quantized, it strongly suggests that some kind of sum over states should reproduce it. In addition, the absence of Newton's constant in (1.1) gives hope that the counting might be performed at small gravitational coupling and then reliably extrapolated to the strong coupling regime where the black hole lies. Identifying the microscopic system behind (1.1) remains an open problem in string theory. This motivates the study of analogous solutions, such as the extremal Myers-Perry (MP) black hole rotating in two independent planes in five dimensions [1], whose entropy

$$
S=2 \pi \sqrt{\left|J_{1} J_{2}\right|}
$$

is closely similar to (1.1), and also of other black holes sharing some of the features of the Kerr solution. In this paper we are interested in having an ergosphere surrounding the horizon.

Recently, a microscopic model for the extremal 5D MP black hole (orbifolded along a certain direction) has been presented, reproducing exactly the entropy (1.2) [2]. The model is based on a connection between the MP solutions and Kaluza-Klein black holes: if we place an MP black hole at the tip of a Taub-NUT geometry we recover a Kaluza-Klein black hole. Since Kaluza-Klein black holes are naturally embedded in Type IIA string theory as solutions with D0 and D6 charges, Ref. [2] used the analysis of

\footnotetext{
*Electronic address: emparan@ub.edu

†Electronic address: sandro@ffn.ub.es
}

D0-D6 bound states in [3] to derive a microscopic model for (1.2).

Kaluza-Klein black holes are also of interest by themselves. In the generic dyonic case they are never supersymmetric, nor are in general close to any supersymmetric state. The entropy of the extremal solutions - with degenerate horizons of zero temperature-depends, like (1.1), only on integer-quantized charges, and not on the coupling or other moduli. There are two branches of extremal black holes, depending on whether their angular momentum is below or above a certain bound [4,5]. Reference [2] developed the statistical description of KK black holes in the slow-rotation regime. In this paper we extend the analysis to show that the entropy of fastly rotating KK black holes can also be accurately reproduced. This is of interest for several reasons. Unlike the slowly-rotating $\mathrm{KK}$ black holes, whose horizons are static, the fastly rotating black holes have nonzero horizon angular velocity, possess ergospheres and exhibit superradiance, so they are qualitatively much closer to the Kerr black hole. In fact, as $J$ grows large with fixed charges, the KK black holes asymptotically approach the Kerr solution. So one may hope for hints for a statistical model of (1.1).

One feature that we find, and which we argue can be expected for the extremal Kerr black hole too, is that the microscopic calculations exactly match the entropy but not the mass of the fastly rotating black hole. As we will see, this fits well with the macroscopic analyses of [6-8] in the context of the attractor mechanism. Our study also gives a clear indication of how extremal rotating black holes with superradiant ergospheres are distinguished microscopically from those that cannot superradiate. Another, perhaps surprising, consequence of our analysis is that both slowly and fastly rotating $\mathrm{KK}$ black holes provide microscopic accounts of the entropy formula (1.2), even if they correspond to rather different microscopic states. As we discuss below, this does not pose any problem, since the microscopic theory always retains a memory of how the 5D black hole is embedded within Taub-NUT. 


\section{EXTREMAL KALUZA-KLEIN BLACK HOLES}

References [4,5] independently constructed the solutions for general Kaluza-Klein dyonic rotating black holes, which we give in appendix A. For more details we refer to these papers and to Ref. [9], which contains insightful remarks on their properties. Here we briefly summarize the most relevant features.

The solutions are characterized by four physical parameters: mass $M$, angular momentum $J$, and electric and magnetic charges $Q$ and $P$. For solutions with a regular horizon, the mass always satisfies

$$
2 G_{4} M \geq\left(Q^{2 / 3}+P^{2 / 3}\right)^{3 / 2} .
$$

The extremal limit, defined as the limit of degenerate, zerotemperature horizon, can be achieved in two ways, giving two distinct branches of solutions:

(i) Slow rotation: $G_{4}|J|<|P Q|$. The mass

$$
2 G_{4} M=\left(Q^{2 / 3}+P^{2 / 3}\right)^{3 / 2}
$$

saturates the bound (2.1) independently of $J$. The angular velocity of the horizon vanishes, and there is no ergosphere. The entropy is

$$
S=2 \pi \sqrt{\frac{P^{2} Q^{2}}{G_{4}^{2}}-J^{2}} .
$$

(ii) Fast rotation: $G_{4}|J|>|P Q|$. The entropy

$$
S=2 \pi \sqrt{J^{2}-\frac{P^{2} Q^{2}}{G_{4}^{2}}}
$$

is the natural continuation of (2.3), but the mass is strictly above the value (2.2) and, for fixed $Q$ and $P$, it grows monotonically with $|J|$. The angular velocity of the horizon is nonzero, and there is an ergosphere.

The extremal horizon disappears and becomes a naked singularity at the dividing value $G_{4}|J|=|P Q|$.

Kaluza-Klein black holes are naturally embedded in Type IIA string theory by taking a product with $T^{6}$. The KK gauge potential is then identified with the RR 1-form potential (so the KK circle is identified with the M theory direction). $Q$ and $P$ correspond to D0 and D6 charges, quantized as

$$
Q=\frac{g}{4 V_{6}} N_{0}, \quad P=\frac{g}{4} N_{6},
$$

where $N_{0}$ and $N_{6}$ are the number of D0 and D6 branes, $g$ is the string coupling, and the volume of $T^{6}$ is $(2 \pi)^{6} V_{6}$. We work in string units, so $G_{4}=g^{2} / 8 V_{6}$.

The mass bound (2.2) becomes

$$
M=\frac{1}{g}\left(N_{0}^{2 / 3}+\left(N_{6} V_{6}\right)^{2 / 3}\right)^{3 / 2},
$$

and the entropies (2.3) and (2.4) become

$$
S=2 \pi \sqrt{\frac{N_{0}^{2} N_{6}^{2}}{4}-J^{2},}
$$

and

$$
S=2 \pi \sqrt{J^{2}-\frac{N_{0}^{2} N_{6}^{2}}{4}},
$$

respectively. In analogy with (1.1), these entropies are independent of $g, V_{6}$ and any other $T^{6}$ moduli.

\section{MICROSCOPIC MODEL OF ROTATING D0-D6 BLACK HOLES}

The microscopic description of two-charge D0-D6 systems requires that we recall first some aspects of fourcharge configurations in Type II string theory compactified on $T^{6}$ (or $\mathrm{M}$ theory on $T^{7}$ ), in particular, when rotation in the noncompact directions is present.

\section{A. Rotating zero-temperature configurations in the $(4,0)$-SCFT}

Consider brane intersections with four charges $N_{1}, N_{2}$, $N_{3}, N_{4}$, in a regime where the dynamics of low energy modes localized at the intersection is described in terms of a chiral $(4,0)$-supersymmetric CFT [10-12]. We shall be somewhat unspecific about what the $N_{i}$ stand for. The statistical entropy counting is most easily performed when $N_{1,2,3}$ denote wrapping numbers of M5 branes, and $N_{4}$ denotes momentum units along the (smoothed) intersection [12]. However, the $U$-dual frame where the $N_{i}$ correspond to four stacks of D3 branes intersecting over a point [13] will be more useful later. Like in [2], the modular invariance of the entropy and angular momentum makes it natural to assume that the degrees of freedom responsible for them are localized at the pointlike intersection.

To recover the SCFT we take the number of antibranes of the 1,2,3 kind to be suppressed, but we allow for both branes (or momentum) and antibranes (or oppositely moving momentum) of type 4. To leading order with $N_{1,2,3} \gg$ 1 , the central charge for both left- and right-moving sectors is $c=6 N_{1} N_{2} N_{3}$, and $L_{0}-\bar{L}_{0}=N_{4}$. Supersymmetric configurations have the left-moving sector in its supersymmetric ground state, $\bar{L}_{0}=N_{L}=0$ [10]. We are, however, interested in exciting the left sector, thus breaking all supersymmetries. The reason is that spacetime rotation requires exciting the fermions in this sector. Their $S U(2)$ $R$-charge acts on spacetime as $S O(3)$ rotation, so a macroscopic angular momentum $J$ results from the coherent polarization of these fermions. This projection also reduces the available phase space, so the effective oscillator number entering the entropy formula is $\tilde{N}_{L}=N_{L}-6 J^{2} / c=$ $N_{L}-J^{2} / N_{1} N_{2} N_{3}$. Then [11] 


$$
\begin{aligned}
S & =2 \pi \sqrt{\frac{c}{6}\left(\sqrt{\tilde{N}_{L}}+\sqrt{N_{R}}\right)} \\
& =2 \pi\left(\sqrt{N_{1} N_{2} N_{3} N_{L}-J^{2}}+\sqrt{N_{1} N_{2} N_{3} N_{R}}\right) .
\end{aligned}
$$

Under the assumption that the constituents interact only very weakly, the total mass of the system is

$$
M=M_{1} N_{1}+M_{2} N_{2}+M_{3} N_{3}+M_{4}\left(N_{R}+N_{L}\right) .
$$

Here $M_{i}$ are the masses of a unit of each single constituent.

Zero-temperature states must have oscillator distributions such that either the left or right "temperatures," $T_{L}$ or $T_{R}$, vanish. For a state with $J \neq 0$, this results into two distinct possibilities:

(i) $T_{R}=0$

Set $N_{R}=0$ and $N_{L}>\frac{J^{2}}{N_{1} N_{2} N_{3}} \geq 0$, so $N_{4}=-N_{L}<$ 0 . The left-moving sector gives rise to both the angular momentum,

$$
J^{2}<N_{1} N_{2} N_{3}\left|N_{4}\right|
$$

and the entropy,

$$
S=2 \pi \sqrt{N_{1} N_{2} N_{3}\left|N_{4}\right|-J^{2}}
$$

Hence the mass

$$
M=M_{1} N_{1}+M_{2} N_{2}+M_{3} N_{3}+M_{4}\left|N_{4}\right|,
$$

is fixed by the charges $N_{i}$ independently of $J$.

(ii) $T_{L}=0$

Set $N_{R}>0, N_{L}=\frac{J^{2}}{N_{1} N_{2} N_{3}}$, so $N_{4}=N_{R}-\frac{J^{2}}{N_{1} N_{2} N_{3}}$. The fermions in the left sector fill up to the Fermi level, so $T_{L}$ is effectively zero. Both sectors are excited, and in principle $N_{4}$ can be either positive, negative, or zero. However, if we require that the right sector be only slightly excited, $N_{R} \ll N_{L}$, then $N_{4}<0$. The left movers provide the angular momentum

$$
J^{2}=N_{1} N_{2} N_{3} N_{L}>N_{1} N_{2} N_{3}\left|N_{4}\right|,
$$

and the right movers the entropy,

$$
S=2 \pi \sqrt{N_{1} N_{2} N_{3} N_{R}}=2 \pi \sqrt{J^{2}-N_{1} N_{2} N_{3}\left|N_{4}\right|} .
$$

From (3.2) we find the mass

$$
\begin{aligned}
M= & M_{1} N_{1}+M_{2} N_{2}+M_{3} N_{3} \\
& +M_{4}\left(N_{4}+2 \frac{J^{2}}{N_{1} N_{2} N_{3}}\right) \\
= & M_{1} N_{1}+M_{2} N_{2}+M_{3} N_{3}+M_{4}\left|N_{4}\right| \\
& +2 M_{4}\left(\frac{J^{2}}{N_{1} N_{2} N_{3}}-\left|N_{4}\right|\right)
\end{aligned}
$$

is strictly above (3.5).
This CFT describes the four-dimensional black holes of $[11,14]$. The restriction to small $N_{R}$, so that $\frac{J^{2}}{N_{1} N_{2} N_{3}}-\left|N_{4}\right|$ is small, is required for the validity of the CFT description. Indeed, it is only in this regime that the supergravity solutions have a locally- $\mathrm{AdS}_{3}$ near-horizon geometry. However, the entropy of the extremal $T_{L}=0$ black holes appears to be correctly reproduced for arbitrary values of the parameters. We will comment more on this in the final section.

\section{B. Microscopics of D0-D6}

According to [3], a system of $N_{0}$ D0 branes bound to $N_{6}$ D6 branes wrapped on $T^{6}$ is $T$-dual to a nonsupersymmetric intersection of four stacks of D3 branes. One of the stacks has reversed orientation relative to the supersymmetric case. This is similar to the configurations of the previous section with $N_{4}<0$ (the supersymmetrybreaking case), but there is one important difference: the D3 branes wrap now nonminimal rational directions $k / l$ in each $T^{2}$ within $T^{6}=T^{2} \times T^{2} \times T^{2}$. The number $N$ of D3 branes is the same in each stack, and

$$
N_{0}=4 k^{3} N, \quad N_{6}=4 l^{3} N
$$

(so $N_{0,6}$ are necessarily multiples of four).

The main assumption of the model, supported by modular invariance, is that the entropy of the low energy excitations at the D3 brane intersection is a local property of the intersection and is independent of whether the branes wrap the torus along minimal or along nonminimal rational cycles. Then we can import the entropy calculations from the previous section by setting

$$
N_{1}=N_{2}=N_{3}=\left|N_{4}\right|=N .
$$

Crucially, we must also take into account that the number of intersection points in the torus does depend on how the branes are wrapped: there are $2 \mathrm{kl}$ intersections in each $T^{2}$, and so a total of $(2 k l)^{3}$ in $T^{6}$. Since the Hilbert space at each intersection is independent of the other intersections, the total entropy is $(2 k l)^{3}$ times the entropy from a single intersection point. The angular momentum is also multiplied by this same factor. Since the total entropy is maximized by distributing $J$ evenly over all intersections, each one carries $J_{0}=J /(2 k l)^{3}$.

In order to obtain the masses for the D3-brane configuration we note that if the volume of minimal 3-cycles in $T^{6}$ is $V_{3}$, then each of the 3-branes has volume $\left(k^{2}+l^{2}\right)^{3 / 2} V_{3}$, so their individual masses are

$$
M_{D 3}=\left(k^{2}+l^{2}\right)^{3 / 2} \frac{V_{3}}{g},
$$

for branes in any of the four stacks.

In this set up, we find that the two different extremal rotating D0-D6 systems of section II map to each of the two zero-temperature rotating intersecting D3-brane sys- 
tems of section III A:

(i) Slow rotation. This was the regime studied in [2]. The CFT at the intersection has the right sector in its ground state, with entropy per intersection given by (3.4). So, for the D0-D6 system,

$$
S_{\text {branes }}=(2 k l)^{3} \times 2 \pi \sqrt{N^{4}-J_{0}^{2}}=2 \pi \sqrt{\frac{N_{0}^{2} N_{6}^{2}}{4}-J^{2}} \text {, }
$$

in exact agreement with (2.7). The mass also matches exactly. Putting $N$ 3-branes with mass (3.11) in each of the four stacks, the total mass is

$$
M_{\text {branes }}=4 N M_{D 3}=\frac{V_{3}}{g}\left(N_{0}^{2 / 3}+N_{6}^{2 / 3}\right)^{3 / 2} .
$$

After $T$-duality in the three appropriate torus directions, the agreement with the mass of the slowrotation D0-D6 black hole (2.6), is exact.

(ii) Fast rotation. We naturally assign to each intersection a state in the fastly rotating regime of the CFT, i.e., $T_{L}=0$. Then, using the entropy formula (3.7),

$$
S_{\text {branes }}=(2 k l)^{3} \times 2 \pi \sqrt{J_{0}^{2}-N^{4}}=2 \pi \sqrt{J^{2}-\frac{N_{0}^{2} N_{6}^{2}}{4}}
$$

we recover the correct value for the D0-D6 black hole (2.8).

The agreement, however, does not extend to the mass in this case. Consider values of $|J|$ slightly above $N_{0} N_{6} / 2$, so there is only a small mass $\delta M>$ 0 above (2.6),

$$
M=\frac{V_{3}}{g}\left(N_{0}^{2 / 3}+N_{6}^{2 / 3}\right)^{3 / 2}+\delta M .
$$

We compute first $\delta M$ within the microscopic brane model. Recall that the mass is simply proportional to the volume of branes of each kind, so we use (3.11) in (3.8). $\delta M$ comes from the last term in (3.8), and we find ${ }^{1}$

$$
\begin{aligned}
\delta M_{\text {branes }} & =\frac{V_{3}}{g}\left(k^{2}+l^{2}\right)^{3 / 2} \times 2\left(\frac{J_{0}^{2}}{N^{3}}-N\right) \\
& =\frac{V_{3}}{g} \frac{\left(N_{0}^{2 / 3}+N_{6}^{2 / 3}\right)^{3 / 2}}{2}\left(\frac{4 J^{2}}{\left(N_{0} N_{6}\right)^{2}}-1\right) .
\end{aligned}
$$

On the other hand, the mass of the black hole gives, after $T$-duality, and to leading order in $\left(J^{2}-\right.$ $\left.\left(N_{0} N_{6}\right)^{2} / 4\right)$,

\footnotetext{
${ }^{1}$ Note that we saturate $N_{L}=J_{0}^{2} / N^{3}$ at each intersection, which is smaller than $J^{2} / N^{3}$.
}

$$
\delta M_{\mathrm{bh}}=\frac{V_{3}}{g} \frac{\left(N_{0} N_{6}\right)^{2 / 3}}{2\left(N_{0}^{2 / 3}+N_{6}^{2 / 3}\right)^{1 / 2}}\left(\frac{4 J^{2}}{\left(N_{0} N_{6}\right)^{2}}-1\right) .
$$

So

$$
\frac{\delta M_{\mathrm{branes}}}{\delta M_{\mathrm{bh}}}=\left[\left(\frac{N_{0}}{N_{6}}\right)^{1 / 3}+\left(\frac{N_{6}}{N_{0}}\right)^{1 / 3}\right]^{2} .
$$

The discrepancy in the masses is naturally attributed to a mass renormalization as the gravitational coupling is increased. Observe that $\delta M_{\text {branes }}>\delta M_{\mathrm{bh}}$, which is as expected since gravitational binding should reduce the energy. In the final section we discuss further why this renormalization occurs for fast but not for slow rotation.

Following the last comments in the previous subsection, in principle it would seem possible to extend the agreement of the entropies to arbitrarily large values of $J^{2} / N_{0}^{2} N_{6}^{2}$, but in these cases the use of the CFT seems largely unjustified. The mass renormalizations get of course much larger.

\section{ERGOSPHERES AND SUPERRADIANCE}

\section{A. Qualitative microscopics}

Extremal rotating black holes with ergospheres provide a clean setting for analyzing superradiance. Since these black holes are at zero temperature, Hawking radiation, which typically mingles with superradiance, is absent. The distinction between the two effects, however, is not sharp: the extremal Kerr black hole does spontaneously emit superradiant modes through quantum effects [15]. This effect drives the black hole from the extremal to a nonextremal state, and superradiant emission smoothly mixes with Hawking radiation.

The statistical description above gives some clear hints of what is the microscopic distinction between extremal rotating black holes with or without ergospheres, and how superradiance arises from the microscopic theory. ${ }^{2}$ Recall first how Hawking radiation appears microscopically. In the 2D CFT, nonextremal, finite-temperature states occur when the effective temperature of the excitations in both left and right sectors is nonzero, with the total system at temperature $T_{H}^{-1}=\left(T_{L}^{-1}+T_{R}^{-1}\right) / 2$. So if we couple the CFT to closed strings that propagate out to the asymptotically flat bulk, then left- and right-moving open string excitations can combine into a closed string, resulting into Hawking radiation at temperature $T_{H}$. If rotation is present, superradiance effects will mix in. However, when one of the sectors is at zero temperature, i.e., at extremality,

\footnotetext{
${ }^{2}$ The following applies not only to Kaluza-Klein black holes but also to the four-charge 4D and three-charge 5D black holes, for which there also exist extremal rotating states with and without ergospheres. Note also that this is quite independent of the presence or absence of unbroken supersymmetry.
} 
thermal Hawking radiation cannot occur. Above we have described two distinct rotating zero-temperature systems. In the first possibility the right-moving sector remains unexcited. So, in the absence of open string excitations of one chirality, there cannot be any closed string emission-neither Hawking emission nor superradiance. This is as it should be, since these states describe extremal black holes without ergospheres.

In contrast, extremal black holes with a superradiating ergosphere correspond to states with both left- and rightmoving excitations. The emission of (nonthermal) closed strings, from the combination of left- and right-moving open string excitations, seems possible now. Moreover, since the left-moving excitations have spin, the emitted closed string will necessarily carry angular momentum away from the black hole. So it is natural to expect that this process describes superradiant emission. The details of this correspondence are being investigated and we hope to report on them elsewhere.

Let us also mention that a different system where Hawking radiation is absent but ergoregions exist is provided by the horizonless smooth rotating solitons of [16]. The fact that these are dual to CFT states ${ }^{3}$ where both the left and right sectors are excited above their Ramond ground states is in agreement with the picture we suggest. These solutions might also provide a convenient setting for the microscopic study of ergoregions.

\section{B. 4D vs 5D perspectives}

The charges $Q$ and $P$, or alternatively the corresponding integers $N_{0}$ and $N_{6}$, have a neat geometrical interpretation from the five-dimensional point of view: $N_{0}$ is the number of units of quantized momentum in the compact fifth direction, and $N_{6}$ is the degree of the fibration of this internal $S^{1}$ on the orbital $S^{2}$ 's. So when $N_{6}>0$ the horizon topology in $5 \mathrm{D}$ is $S^{3} / \mathbb{Z}_{N_{6}}$. If the horizon size is much smaller than the compact radius, the black hole can be regarded as an MP black hole at the tip of a Taub-NUT geometry [17]. We elaborate in detail on this in appendix A, and mention here only some salient features.

The MP black hole generically has angular momenta $J_{1}$, $J_{2}$ in two independent rotation planes. The KK electric charge $Q$ is proportional to the self-dual component of the angular momentum, $\mathcal{J}=J_{1}+J_{2}$, aligned with the KK fiber, and $J$ to the anti-self-dual component $\overline{\mathcal{J}}=J_{1}-J_{2}$, off the KK direction. In the extremal limit, the MP black hole entropy reduces to (1.2), which can be written as

$$
S=\pi \sqrt{\left|\mathcal{J}^{2}-\overline{\mathcal{J}}^{2}\right|}
$$

Both the slowly and the fastly rotating extremal KK black holes lead, in the decompactification limit, to extremal MP black holes, the former with $\mathcal{J}^{2}>\overline{\mathcal{J}}^{2}$, the latter

\footnotetext{
${ }^{3}$ In this case, the $(4,4)-$ SCFT of the D1-D5 system.
}

with $\mathcal{J}^{2}<\overline{\mathcal{J}}^{2}$. From a purely 5D (decompactified) viewpoint, this distinction is obviously arbitrary. However, we have found that the brane configurations describing each of these two regimes are rather different. The point is that the symmetry between $\mathcal{J}$ and $\overline{\mathcal{J}}$ is broken once we put the MP black hole at a certain orientation within Taub-NUT. There is a choice to be made of which of the $5 \mathrm{D}$ angular momenta is going to correspond to the four-dimensional $J$ and which to $Q$. So the two microscopic configurations actually describe two different ways to embed the extremal MP black holes within Taub-NUT, and in this sense they describe different black holes.

One might then ask how it can be that the MP black hole in Taub-NUT is capable of superradiating when $\mathcal{J}^{2}<\overline{\mathcal{J}}^{2}$, but not when $\mathcal{J}^{2}>\overline{\mathcal{J}}^{2}$. It turns out that, as we show in detail in appendix B, superradiance is possible in both situations but is interpreted differently in each case. Consider an incident wave in the KK black hole background with dependence

$$
\Psi \sim e^{i k y+i n \phi-i \omega t}
$$

on the Killing directions, $y$ being the coordinate along the $\mathrm{KK}$ circle. The wavenumber $k$ is KK electric charge from the 4D viewpoint. We find that the necessary condition for superradiant amplification is

$$
k<\omega<n \Omega_{H}+2 G_{4} k \Phi_{E}
$$

where $\Omega_{H}$ is the $4 \mathrm{D}$ horizon angular velocity and $\Phi_{E}$ is the $\mathrm{KK}$ electric potential. From the $5 \mathrm{D}$ viewpoint, $2 G_{4} \Phi_{E}$ is the velocity at which the 5D horizon is rotating in the $y$ direction relative to static asymptotic observers.

The conventional rotational superradiance of fastly rotating $\mathrm{KK}$ black holes corresponds to amplification of neutral $(k=0)$ waves with $\omega<n \Omega_{H}$. We show in appendix B that this is indeed possible for scalar fields. This is the process whose microscopic dual is suggested in the previous section.

On the other hand, slowly spinning extremal black holes have $\Omega_{H}=0$ so they show no rotational superradiance. But they can produce superradiant amplification of waves with KK electric charge $k$. In appendix B we show that this indeed happens and is always allowed since these black holes have $2 G_{4} \Phi_{E}>1$. This process, however, is not so naturally described in the dual CFT system, since it requires either the emission of 4D charge and hence changing the central charge of the CFT, or altering the direction in which the branes wrap $T^{6}$, which is not seen by the CFT.

\section{DISCUSSION}

We have shown that it is possible to successfully extend the microscopic model of KK black holes in [2] to cover the regime of fast rotation, with horizons that rotate with nonvanishing angular velocity and therefore are more similar to the Kerr black hole. There exist other similar instances where the entropy is also correctly reproduced: 
extremal four-charge type II black holes also have slow and fast rotation regimes which are correctly captured by the CFT of Sec. III A, and there are analogous three-charge five-dimensional black holes with these properties which can be described in the $(4,4)$-SCFT of the D1-D5 system $[11,14]$. However, in these cases not only the entropy but also the mass is accurately reproduced by the microscopic model, both at slow and at fast rotation (at least for rotation slightly above the divide). This agreement is understood, within the context of AdS/CFT duality, as being due to the existence of a locally $\mathrm{AdS}_{3}$ (BTZ) geometry near the horizon [18,19]. In contrast, extremal KK black holes do not have in general $\mathrm{AdS}_{3}$ symmetry near the horizon (only in the singular case $|P Q|=G_{4}|J|$, i.e., when either $J_{1}$ or $J_{2}$ vanish, do they have in $5 \mathrm{D}$ the near-horizon symmetry $S O(2,2)$ of $\left.\mathrm{AdS}_{3}[20]\right),{ }^{4}$ so perhaps we should be surprised by the fact that the entropy does come out correctly.

Actually, our results are in perfect agreement with the recent macroscopic studies in [6-8], which argue that the $S L(2, \mathbb{R}) \times U(1)$ symmetry near the horizon of fourdimensional extremal rotating black holes, charged as well as neutral, ensures that the macroscopic value for the entropy can be extrapolated to weak coupling. Extremal KK black holes do possess this near-horizon symmetry (and their MP limits too [20]). Hence, if a microscopic model is identified, its entropy should exactly match the macroscopic value. In this paper we have provided this microscopic model and confirmed the agreement of entropies.

Reference [6] finds that the scalar field, and indeed the whole solution, for slowly rotating KK black holes is attracted to a completely fixed form near the horizon, so not only the entropy but also the mass is fixed-in agreement with the microscopic calculation in [2]. However, for fastly rotating extremal KK black holes there exist flat directions in the effective potential for the scalar near the horizon, with the effect that only the entropy is attracted to a fixed value. Other quantities, like the mass, are not guaranteed to be fixed. We have found that in fact they are not. Thus we conclude that the attractor mechanism correctly predicts which quantities will match at weak and strong coupling, and which ones will, generically, be renormalized.

Extremal fastly rotating four-charge black holes with $J^{2} \gg N_{1} N_{2} N_{3}\left|N_{4}\right|$, and KK black holes with $J^{2} \gg$ $N_{0}^{2} N_{6}^{2}$, do have only $S L(2, \mathbb{R}) \times U(1)$ near-horizon symmetry. In principle these black holes can approach arbitrarily closely to the extremal Kerr solution. Their entropies, but not their masses, agree with naive CFT formulas, although one is far from the regime where any application of the CFT is justified. So, even if this may not

\footnotetext{
${ }^{4}$ This seems to be related to the fact that the solution can be reached as a limit of a black ring [21]. It is also analogous to the phenomenon discussed for the BMPV black hole in [22,23].
}

be the correct description, it seems likely that a microscopic model for the extremal Kerr solution, which also has near-horizon symmetry $S L(2, \mathbb{R}) \times U(1)$ [20], should be able to pin down exactly the entropy (1.1), but not the mass of the black hole. Obtaining the exact entropy of nonextremal vacuum black holes, like Schwarzschild, will require taking into account mass renormalization effects.

\section{ACKNOWLEDGMENTS}

We thank Gary Horowitz for comments on an earlier version of the manuscript. This work was supported in part by DURSI 2005 SGR 00082, CICYT FPA 2004-04582C02-02 and EC FP6 program MRTN-CT-2004-005104. A. M. was partially supported by a FPU grant from MEC (Spain).

\section{APPENDIX A: MYERS-PERRY FROM KALUZA-KLEIN: GENERAL CASE}

From a five-dimensional standpoint, KK black holes with nonzero magnetic charge can be regarded as black holes sitting at the tip of a Taub-NUT space, at least as long as the black hole size is much smaller than the compact radius. This was noted, in a particular case, in Ref. [17], which showed that in a limit of large fifth-dimensional radius the static $(J=0)$ dyonic Kaluza-Klein black holes reduce to five-dimensional Myers-Perry black holes with self-dual angular momentum. This corresponds to the case where the rotation of the MP black hole is aligned exactly along the Kaluza-Klein direction. In this appendix we consider the most general case: the KK black hole has nonvanishing four-dimensional angular momentum $J$, which corresponds to the anti-self-dual component of the angular momentum of the MP black hole. We keep parameters in the KK solution arbitrary, in particular, we do not confine ourselves to extremal limits. Hence we are able to recover the general MP solution.

\section{Limiting procedure}

We write the solution in essentially the form given in [5], and use the results therein for the physical parameters. ${ }^{5}$ In five-dimensional form,

$$
\begin{aligned}
d s^{2}= & \frac{H_{q}}{H_{p}}(d y+\mathbf{A})^{2}-\frac{\Delta_{\theta}}{H_{q}}(d t+\mathbf{B})^{2} \\
& +H_{p}\left(\frac{d r^{2}}{\Delta}+d \theta^{2}+\frac{\Delta}{\Delta_{\theta}} \sin ^{2} \theta d \phi^{2}\right),
\end{aligned}
$$

where

\footnotetext{
${ }^{5}$ Here, and in (A21) below, our sign choices for the rotation parameters are such that positive $\alpha, a$, and $b$ correspond to positive rotation.
} 


$$
\begin{gathered}
H_{p}=r^{2}+\alpha^{2} \cos ^{2} \theta+r(p-2 m)+\frac{p}{p+q} \frac{(p-2 m)(q-2 m)}{2}+\frac{p}{2 m(p+q)} \sqrt{\left(q^{2}-4 m^{2}\right)\left(p^{2}-4 m^{2}\right)} \alpha \cos \theta, \\
H_{q}=r^{2}+\alpha^{2} \cos ^{2} \theta+r(q-2 m)+\frac{q}{p+q} \frac{(p-2 m)(q-2 m)}{2}-\frac{q}{2 m(p+q)} \sqrt{\left(q^{2}-4 m^{2}\right)\left(p^{2}-4 m^{2}\right)} \alpha \cos \theta, \\
\Delta_{\theta}=r^{2}+\alpha^{2} \cos ^{2} \theta-2 m r, \\
\Delta=-\left[2 Q\left(r+\frac{p-2 m}{2}\right)-\sqrt{\frac{q^{3}\left(p^{2}-4 m^{2}\right)}{4 m^{2}(p+q)}} \alpha \cos \theta\right] H_{q}^{-1} d t \\
-\left[2 P\left(H_{q}+\alpha^{2} \sin ^{2} \theta\right) \cos \theta-\sqrt{\frac{p\left(q^{2}-4 m^{2}\right)}{4 m^{2}(p+q)^{3}}}\left[(p+q)\left(p r-m(p-2 m)+q\left(p^{2}-4 m^{2}\right)\right] \alpha \sin ^{2} \theta\right] H_{q}^{-1} d \phi,\right. \\
\mathbf{B}=\sqrt{p q} \frac{\left(p q+4 m^{2}\right) r-m(p-2 m)(q-2 m)}{2 m(p+q) \Delta_{\theta}} \alpha \sin ^{2} \theta d \phi .
\end{gathered}
$$

The (four-dimensional) physical parameters are

$$
\begin{gathered}
2 G_{4} M=\frac{p+q}{2}, \\
G_{4} J=\frac{\sqrt{p q}\left(p q+4 m^{2}\right)}{4(p+q)} \frac{\alpha}{m}, \\
Q^{2}=\frac{q\left(q^{2}-4 m^{2}\right)}{4(p+q)}, \\
P^{2}=\frac{p\left(p^{2}-4 m^{2}\right)}{4(p+q)} .
\end{gathered}
$$

Solutions with black hole horizons have $q \geq 2 m, p \geq 2 m$, $m \geq|\alpha|$.

For regularity, the coordinate $y$ must be periodically identified as

$$
y \sim y+2 \pi R, \quad R=\frac{4 P}{N_{6}},
$$

for integer $N_{6}$. As usual, $\phi \sim \phi+2 \pi$. From a fivedimensional viewpoint the KK electric charge is momentum along the $y$-direction and so is quantized as

$$
Q=\frac{2 G_{4} N_{0}}{R}
$$

for integer $N_{0}$. In the string theory embedding, $N_{0}$ and $N_{6}$ correspond to the numbers of D0 and D6 branes introduced in (2.5), and $R=g$ in string units.

We take a limit where the magnetic charge $P$ grows to infinity while $Q, J$ and the black hole size remain finite. This has the effect of effectively decompactifying the fifth direction. To perform this, we send $p \rightarrow \infty$, and also send $r, m, \alpha, q \rightarrow 0$, and $y \rightarrow \infty$ in such a way that $p r, p m, p \alpha$, $p q, y / p$, remain finite. It is convenient to introduce new finite parameters $\mu, a, b$, and finite radial and angular coordinates, $\rho$ and $\psi$, through

$$
\begin{gathered}
p q=\frac{\mu}{4}, \\
p \alpha=\frac{1}{8}\left(\mu-(a+b)^{2}\right)^{1 / 2}(a-b), \\
p m=\frac{1}{8}\left[\mu\left(\mu-(a+b)^{2}\right)\right]^{1 / 2}, \\
p r=\frac{1}{4}\left[\rho^{2}-\frac{1}{2}\left(\mu-a^{2}-b^{2}-\sqrt{\mu\left(\mu-(a+b)^{2}\right)}\right),\right. \\
\psi=\frac{y}{p}, \quad \text { with } \quad \psi \sim \psi+\frac{4 \pi}{N_{6}} .
\end{gathered}
$$

The angles $(\psi, \phi, \theta)$ are Euler angles for (a topological) $S^{3} / \mathbb{Z}_{N_{6}}$. It is also convenient to use

$$
\tilde{\psi}=\frac{\psi+\phi}{2}, \quad \tilde{\phi}=\frac{\psi-\phi}{2}, \quad \tilde{\theta}=\frac{\theta}{2},
$$

with

$$
(\tilde{\psi}, \tilde{\phi}) \sim\left(\tilde{\psi}+\frac{2 \pi}{N_{6}}, \tilde{\phi}+\frac{2 \pi}{N_{6}}\right) \sim(\tilde{\psi}, \tilde{\phi}+2 \pi) .
$$

After lengthy algebra, in the limit $p \rightarrow \infty$ the metric (A1) becomes 


$$
\begin{aligned}
d s^{2}= & -d t^{2}+\frac{\mu}{\Sigma}\left(d t-a \sin ^{2} \tilde{\theta} d \tilde{\psi}-b \cos ^{2} \tilde{\theta} d \tilde{\phi}\right)^{2} \\
& +\Sigma\left(\frac{d \rho^{2}}{\tilde{\Delta}}+d \tilde{\theta}^{2}\right)+\left(\rho^{2}+a^{2}\right) \sin ^{2} \tilde{\theta} d \tilde{\psi}^{2} \\
& +\left(\rho^{2}+b^{2}\right) \cos ^{2} \tilde{\theta} d \tilde{\phi}^{2},
\end{aligned}
$$

with

$$
\begin{gathered}
\Sigma=\rho^{2}+a^{2} \cos ^{2} \tilde{\theta}+b^{2} \sin ^{2} \tilde{\theta}, \\
\tilde{\Delta}=\frac{\left(\rho^{2}+a^{2}\right)\left(\rho^{2}+b^{2}\right)-\mu \rho^{2}}{\rho^{2}} .
\end{gathered}
$$

This is the general five-dimensional MP black hole, with independent rotation parameters $a$ and $b$ [1]. When $N_{6}>1$ the orbifold identification (A18) implies that the solution is not globally asymptotically flat, but instead the spatial geometry asymptotes to $\mathbb{R}^{4} / \mathbb{Z}_{N_{6}}$. In this case the MP black hole sits at the tip of a conical space.

\section{Relations between physical parameters}

The 4D and 5D Newton constants are as usual related by

$$
G_{4}=\frac{G_{5}}{2 \pi R},
$$

with $R$ given in (A12).

The 4D mass, given by (A8), is dominated in the limit $p \rightarrow \infty$ by the magnetic KK monopole mass. This is identified as

$$
\mathcal{M}_{\mathrm{KK}}=\frac{P}{2 G_{4}},
$$

and diverges as $p \rightarrow \infty$. The finite limiting difference between the total $4 \mathrm{D}$ mass and the KK monopole mass corresponds exactly to the $5 \mathrm{D}$ mass,

$$
M-\mathcal{M}_{\mathrm{KK}} \rightarrow \frac{3 \pi}{8 N_{6} G_{5}} \mu=M_{(5)} .
$$

According to this equation, we can regard the 5D mass as the excitation energy above the KK monopole background. The $N_{6}$ in the denominator comes from integration over the $\mathbb{Z}_{N_{6}}$-orbifolded $S^{3}$.

Consider the following two Killing vectors of the KK black hole geometry,

$$
\zeta_{(1),(2)}=2 P \partial_{y} \pm \partial_{\phi} .
$$

Their associated conserved charges are ${ }^{6}$

$$
J_{1,2}=\frac{P Q}{G_{4}} \pm J=\frac{N_{0} N_{6}}{2} \pm J,
$$

which are independent of $R$ and therefore remain invariant as $p \rightarrow \infty$. In this limit

\footnotetext{
${ }^{6}$ Our definitions of $J_{1,2}$ differ from [2] by a factor of $N_{6}$.
}

$$
\zeta_{(1)} \rightarrow \partial_{\tilde{\psi}}, \quad \zeta_{(2)} \rightarrow \partial_{\tilde{\phi}},
$$

so $J_{1}$ and $J_{2}$ become the angular momenta of the MP black hole in the directions $\tilde{\psi}$ and $\tilde{\phi}$,

$$
J_{1} \rightarrow \frac{\pi \mu a}{4 G_{5} N_{6}}, \quad J_{2} \rightarrow \frac{\pi \mu b}{4 G_{5} N_{6}} .
$$

So, from (A28), the electric charge, which is the component of the rotation aligned with the KK direction $y$, corresponds to the 5D self-dual angular momentum $\mathcal{J}$

$$
N_{0}=\frac{J_{1}+J_{2}}{N_{6}}=\frac{\mathcal{J}}{N_{6}},
$$

and the $4 \mathrm{D}$ angular momentum,

$$
J=\frac{J_{1}-J_{2}}{2}=\frac{\overline{\mathcal{J}}}{2},
$$

is the 5D anti-self-dual angular momentum $\overline{\mathcal{J}}$. This is a $U(1)$ charge in the $S U(2) \subset S O(4) / \mathbb{Z}_{N_{6}}$ that remains unbroken by the compactification. It is the component of the rotation of the MP black hole that lies away from the compactification direction.

It can also be checked, with some work, that the entropy measured from the area in four dimensions agrees in the limit with the entropy of the MP black hole,

$$
\begin{aligned}
S & =\frac{\mathcal{A}_{\mathrm{KKbh}}}{4 G_{4}}=\frac{\pi \sqrt{p q}}{G_{4}}\left[m+\frac{p q+4 m^{2}}{2 m(p+q)} \sqrt{m^{2}-\alpha^{2}}\right] \\
& \rightarrow \frac{2 \pi^{2}}{4 G_{5} N_{6}} \mu \rho_{+}=\frac{\mathcal{A}_{\mathrm{MPbh}}}{4 G_{5}}
\end{aligned}
$$

(with $\rho_{+}$the outer horizon radius).

\section{The two extremal limits}

There are two different extremal limits for KK black holes, which correspond to two different classes of extremal limit for the MP black holes:

(i) Slowly-rotating extremal $\mathrm{KK}$ black holes are the limit of (A1) where $\alpha, m \rightarrow 0$ with finite $|\alpha| / m<$ 1. This implies $G_{4}|J|<|P Q|$.

In the decompactification limit to the MP black hole, this corresponds to

$$
\mu=(a+b)^{2} \quad \text { with } \quad a b>0,
$$

which is

$$
M_{(5)}^{3}=\frac{27 \pi}{32 G_{5} N_{6}} \mathcal{J}^{2}, \quad \text { with } \quad|\mathcal{J}|>|\overline{\mathcal{J}}| .
$$

(ii) Fastly rotating extremal KK black holes have $|\alpha|=$ $m>0$, so $G_{4}|J|>|P Q|$. In the decompactification limit this is

$$
\mu=(a-b)^{2} \quad \text { with } \quad a b<0,
$$




$$
\begin{aligned}
& \text { i.e., } \\
& \qquad M_{(5)}^{3}=\frac{27 \pi}{32 G_{5} N_{6}} \overline{\mathcal{J}}^{2}, \quad \text { with } \quad|\overline{\mathcal{J}}|>|\mathcal{J}| .
\end{aligned}
$$

The mass bound (2.1) translates into a bound on $M_{(5)}$ in terms of $\mathcal{J}$,

$$
M_{(5)}^{3}=\frac{27 \pi}{32 G_{5} N_{6}} \mathcal{J}^{2},
$$

which is obviously saturated at extremal slow-rotation, (A35), and never saturated at fast rotation, (A37). From a purely $5 \mathrm{D}$ viewpoint the distinction is arbitrary. It is only when we put the solution at a certain orientation within Taub-NUT that the symmetry between $\mathcal{J}$ and $\overline{\mathcal{J}}$ is broken.

Finally, since

$$
J_{1} J_{2}=\frac{\left(N_{0} N_{6}\right)^{2}}{4}-J^{2},
$$

we can write the entropy as

$$
S=2 \pi \sqrt{\left|J_{1} J_{2}\right|},
$$

independently of $R$. The two extremal cases above correspond to $J_{1} J_{2}>0$ and $J_{1} J_{2}<0$, respectively.

\section{APPENDIX B: ERGOSPHERES AND SUPERRADIANCE IN EXTREMAL KK BLACK HOLES}

Let us first consider a general necessary condition for superradiant scattering, which follows from the second law of black hole thermodynamics. From the 4D point of view, we must have

$$
T_{H} d S=d M-\Phi_{E} d Q-\Phi_{M} d P-\Omega_{H} d J>0
$$

(the condition is still valid in the extremal limit where $T_{H} \rightarrow 0$ ). We only consider processes where the topology of the 5D solution remains fixed, so $d P=0$.

Consider a scalar field $\Psi$ in the black hole background (A1), satisfying

$$
\square_{(5)} \Psi=0
$$

with the form

$$
\Psi=\psi_{\omega k n}(r, \theta) e^{i k y+i n \phi-i \omega t} .
$$

Here $k$ is interpreted as KK electric charge and is quantized in units of $1 / R$. From a $4 \mathrm{D}$ viewpoint it also gives a rest mass, so if the charged wave is to propagate to infinity it must satisfy $\omega>|k|$.

It can easily be shown that absorption of this field by the black hole results in a change in black hole parameters such that

$$
\frac{\delta J}{\delta M}=\frac{n}{\omega}, \quad \frac{\delta Q}{\delta M}=2 G_{4} \frac{k}{\omega} .
$$

Then (B1) requires

$$
\delta M\left(1-\frac{n}{\omega} \Omega_{H}-2 G_{4} \frac{k}{\omega} \Phi_{E}\right)>0 .
$$

Since we are considering a process of energy extraction, $\delta M<0$, the only way for this to hold is that

$$
\omega<n \Omega_{H}+2 G_{4} k \Phi_{E} .
$$

(i) For the slowly-rotating extremal solution the fourdimensional horizon has $\Omega_{H}=0$ and hence what we have is a charge ergosphere. We can only extract energy by discharging the black hole. The electric potential for these black holes is

$$
2 G_{4} \Phi_{E}=\sqrt{\frac{p+q}{q}}>1
$$

so the charge-superradiance condition can be satisfied.

(ii) The fastly rotating extremal solution has both nonzero angular velocity and electric potential on the horizon,

$$
\Omega_{H}=\frac{1}{\sqrt{p q}}
$$

and

$$
2 G_{4} \Phi_{E}=\sqrt{\frac{q^{2}-4 m^{2}}{q(p+q)}}<1 .
$$

Rotational superradiance of neutral $(k=0)$ waves is obviously possible. Bearing in mind that $\omega>k$ for a wave to escape, then it is not possible to extract energy by simply discharging the black hole $(k>0$, $n=0$ ), but it seems possible to do so by simultaneous extraction of angular momentum and charge.

Note that (B6) is necessary, but not sufficient, for superradiance to be possible. Next we perform a detailed analysis of scalar wave propagation to find, in particular, illustrative cases, that superradiance indeed happens when this is satisfied. We consider extremal black holes with nonzero magnetic charge, but set to zero either $J$ or $Q$, to obtain simple examples of slowly and fastly rotating black holes.

\section{1. $Q \neq \mathbf{0}, J=\mathbf{0}$ extremal black hole}

This is the static (in 4D) limit of slowly-rotating black holes, obtained taking $\alpha=0$ and then $m \rightarrow 0$. The horizon is at $r=0$ and from a 5D viewpoint it is moving along $y$. Indeed, the horizon is generated by orbits of the Killing vector 


$$
\xi=\frac{\partial}{\partial t}+v_{H} \frac{\partial}{\partial y}
$$

where

$$
v_{H}=\sqrt{\frac{p+q}{q}} .
$$

So the horizon is rotating at velocity $v_{H}$ relative to asymptotic static observers that follow orbits of $\partial_{t}$. The vector $\partial_{t}$ becomes spacelike for $r<r_{e}=\frac{1}{2}\left(q-p+\sqrt{q^{2}+p^{2}}\right)$, so there is an ergosphere, which from the $4 \mathrm{D}$ viewpoint is a charge ergosphere. The velocity $v_{H}$ is actually the same as the KK electric potential $2 G_{4} \Phi_{E}$. The fact that $v_{H}>1$ does not result in any causal pathology.

We now analyze if there are massless scalar superradiant modes in this background. The Eq. (B2) is separable for the ansatz

$$
\Psi=\frac{f(r)}{\chi(r)} \Theta(\theta) e^{i n \phi+i k y-i \omega t}
$$

where

$$
\begin{aligned}
\chi(r)= & {\left[\left(2 q r^{2}+2 p r(q+r)\right.\right.} \\
& +p(q+2 r))(2 q r(q+r) \\
& \left.\left.+p\left(q^{2}+2 q r+2 r^{2}\right)\right)\right]^{1 / 4} .
\end{aligned}
$$

For the angular part we get

$$
\begin{aligned}
& \frac{1}{\sin \theta} \frac{d}{d \theta}\left(\sin \theta \frac{d \Theta}{d \theta}\right) \\
& +\left(\lambda_{\text {lnk }}+\frac{1}{\sin ^{2} \theta}\left(p k \sqrt{\frac{p}{p+q}}+n \cos \theta\right)^{2}\right) \Theta=0
\end{aligned}
$$

where $\lambda_{l n k}$ is a separation constant.

For the radial part we obtain

$$
\frac{d^{2} f}{d r_{*}^{2}}+V(r) f=0
$$

where we have defined the "tortoise" radial coordinate $r_{*}$ as

$$
\begin{aligned}
\frac{d r_{*}}{d r}= & \frac{1}{2 r^{2}(p+q)}\left(p^{3} q^{3}+4 p^{2} q^{2}(p+q) r\right. \\
& +6 p q(p+q)^{2} r^{2}+4(p+q)^{3} r^{3} \\
& \left.+4(p+q)^{2} r^{4}\right)^{1 / 2}
\end{aligned}
$$

and whose asymptotic behavior is

$$
\begin{cases}r_{*} \sim r & \text { for } r \rightarrow \infty \\ r_{*} \sim-\frac{1}{r} & \text { for } r \rightarrow 0\end{cases}
$$

For the analysis of superradiance, we follow the approach of [24], which only requires the asymptotic behavior of (B15). Near the horizon

$$
V(r) \simeq \omega_{H}^{2}+O(r) \quad(r \rightarrow 0)
$$

with

$$
\omega_{H}=\omega-v_{H} k
$$

Near infinity

$$
V(r) \simeq \omega_{\infty}^{2}+O(1 / r) \quad(r \rightarrow \infty),
$$

where $\omega_{\infty}^{2}=\omega^{2}-k^{2}$. Then

$$
f(r) \sim \begin{cases}e^{-i \omega_{\infty} r_{*}}+R e^{i \omega_{\infty} r_{*}}, & r \rightarrow \infty \\ T e^{-i \omega_{H} r_{*}}, & r \rightarrow 0,\end{cases}
$$

is a wave of unitary amplitude traveling from infinity and then splitting into a transmitted wave of amplitude $T$ that goes into the horizon, and a reflected wave of amplitude $R$ which goes back to infinity. If (B21) corresponds to a solution of (B15) so does its complex conjugate

$$
f^{*}(r) \sim \begin{cases}e^{i \omega_{\infty} r_{*}}+R^{*} e^{-i \omega_{\infty} r_{*}}, & r \rightarrow \infty \\ T^{*} e^{i \omega_{H} r_{*}}, & r \rightarrow 0 .\end{cases}
$$

These two solutions are linearly independent, and the theory of ordinary differential equations tells us that their Wronskian $W=f f^{*}-f^{*} f^{\prime}$ must be independent of $r$. Near infinity this is $W=2 i \omega_{\infty}\left(|R|^{2}-1\right)$, and near the horizon $W=-2 i \omega_{H}|T|^{2}$. Equating these we get

$$
|R|^{2}=1-\frac{\omega_{H}}{\omega_{\infty}}|T|^{2} .
$$

A wave traveling from and to infinity $(\omega>|k|)$ will undergo superradiant amplification $(|R|>1)$ if $\omega_{H}<0$, i.e.,

$$
k<\omega<v_{H} k .
$$

This can be always fulfilled since $v_{H}>1$. Since these black holes have $\Omega_{H}=0$, it reproduces correctly the condition (B6).

\section{2. $Q=0, J \neq 0$ extremal black hole}

This is a particular case of extremal fastly rotating black holes. It is obtained taking $|\alpha|=m$ and $q=2 m$. The latter sets $Q=0$.

Now the horizon is at $r=m$. The ergosphere is a squashed sphere given by $r=m(1+\sin \theta)$, which touches the horizon at $\theta=0$. The Killing horizon generator is

$$
\xi=\frac{\partial}{\partial t}+\Omega_{H} \frac{\partial}{\partial \phi}
$$

where

$$
\Omega_{H}=\frac{1}{\sqrt{2 m p}} .
$$

The procedure to study superradiance is as in the previous section. For simplicity we consider a field without any dependence on $y$, so in 4D terms this is an electrically neutral $(k=0)$ scalar field. Since the background is also 
neutral, the only effect of having $k \neq 0$ would be to give a $4 \mathrm{D}$ mass to the field.

The Klein-Gordon equation is separable for an ansatz of the form

$$
\Psi=\frac{f(r)}{\chi(r)} \Theta(\theta) e^{i n \phi-i \omega t}
$$

with

$$
\chi(r)=\left[\left(m^{2}+r^{2}\right)\left(m^{2}-2 m r+r(p+r)\right)\right]^{1 / 4} .
$$

The angular equation is

$$
\frac{1}{\sin \theta} \frac{d}{d \theta}\left(\sin \theta \frac{d \Theta}{d \theta}\right)+\left(\lambda_{l n}+m^{2} \omega^{2} \cos ^{2} \theta-\frac{n^{2}}{\sin ^{2} \theta}\right) \Theta=0
$$

which is the equation for spheroidal harmonics. The radial equation takes again the form (B15), but with a different $V(r)$ and a different tortoise coordinate, now defined as

$$
\frac{d r_{*}}{d r}=\frac{1}{(r-m)^{2}}\left[\left(m^{2}+r^{2}\right)\left(m^{2}-2 m r+r(p+r)\right)\right]^{1 / 2}
$$

and with the asymptotic behavior

$$
\begin{cases}r_{*} \sim r & \text { for } r \rightarrow \infty \\ r_{*} \sim-\frac{1}{r-m} & \text { for } r \rightarrow m .\end{cases}
$$

The potential goes as

$$
V(r) \rightarrow \begin{cases}\omega^{2} & \text { for } r \rightarrow \infty \\ \left(\omega-\Omega_{H} n\right)^{2} & \text { for } r \rightarrow m .\end{cases}
$$

In this case we have no potential barrier near infinity from the KK masses. Arguing as before, we get superradiant modes for

$$
0<\omega<\Omega_{H} n
$$

\section{3. $Q \neq \mathbf{0}, J \neq \mathbf{0}$, fastly rotating extremal black hole}

The generalization from the previous section to fastly rotating black holes with $Q \neq 0(\alpha=m, q>2 m)$ is straightforward but very cumbersome, so we do not provide here the full calculation. The result one obtains is the natural generalization of the electrically neutral case: (B33) is still valid, now with the general value (B8) for $\Omega_{H}$. Since we are considering neutral fields, $k=0$, this is in perfect agreement with (B6).
[1] R. C. Myers and M. J. Perry, Ann. Phys. (N.Y.) 172, 304 (1986).

[2] R. Emparan and G. T. Horowitz, Phys. Rev. Lett. 97, 141601 (2006).

[3] W. I. Taylor, Nucl. Phys. B508, 122 (1997).

[4] D. Rasheed, Nucl. Phys. B454, 379 (1995).

[5] F. Larsen, Nucl. Phys. B575, 211 (2000).

[6] D. Astefanesei, K. Goldstein, R. P. Jena, A. Sen, and S. P. Trivedi, J. High Energy Phys. 10 (2006) 058.

[7] A. Dabholkar, A. Sen, and S. Trivedi, J. High Energy Phys. 01 (2007) 096.

[8] D. Astefanesei, K. Goldstein, and S. Mahapatra, hep-th/ 0611140.

[9] F. Larsen, hep-th/0002166.

[10] J. M. Maldacena and A. Strominger, Phys. Rev. Lett. 77, 428 (1996); C. V. Johnson, R. R. Khuri, and R. C. Myers, Phys. Lett. B 378, 78 (1996).

[11] G. T. Horowitz, D. A. Lowe, and J. M. Maldacena, Phys. Rev. Lett. 77, 430 (1996).

[12] J. M. Maldacena, A. Strominger, and E. Witten, J. High Energy Phys. 12 (1997) 002.
[13] V. Balasubramanian and F. Larsen, Nucl. Phys. B478, 199 (1996).

[14] M. Cvetic and D. Youm, Phys. Rev. D 54, 2612 (1996).

[15] D. N. Page, Phys. Rev. D 14, 3260 (1976).

[16] V. Jejjala, O. Madden, S. F. Ross, and G. Titchener, Phys. Rev. D 71, 124030 (2005).

[17] N. Itzhaki, J. High Energy Phys. 09 (1998) 018.

[18] M. Cvetic and F. Larsen, Nucl. Phys. B531, 239 (1998); Phys. Rev. Lett. 82, 484 (1999).

[19] For a review, see P. Kraus, hep-th/0609074.

[20] J.M. Bardeen and G. T. Horowitz, Phys. Rev. D 60, 104030 (1999).

[21] R. Emparan and H. S. Reall, Phys. Rev. Lett. 88, 101101 (2002); for a review, see Classical Quantum Gravity 23, R169 (2006).

[22] N. Alonso-Alberca, E. Lozano-Tellechea, and T. Ortín, Classical Quantum Gravity 20, 423 (2003).

[23] M. Guica and A. Strominger, hep-th/0701011.

[24] A. A. Starobinskii, Sov. Phys. JETP 37, 28 (1973). 